\title{
An unusual case of trapped ovary in a peritoneal pouch causing extrinsic ureteric compression associated with endometriosis
}

Published online: 13 December 2005

C) Springer-Verlag Berlin/Heidelberg 2005

\begin{abstract}
A 35-year-old woman presented with longstanding right loin to groin pain and a right ovarian cyst. The ovarian cyst was considered physiological at the initial gynaecological evaluation. A subsequent transvaginal scan demonstrated a cystic immobile ovary adherent to the pelvic side wall. Laparoscopy revealed endometriosis and a peritoneal defect holding the cystic ovary against the ureter and causing compression with secondary loin pain. The trapped ovary was removed and the patient was cured of the long-standing ureteric colic. This is the first reported case of extrinsic ureteric compression caused by trapped ovary in a peritoneal pouch associated with endometriosis. The diagnosis was suspected preoperatively from the transvaginal scan findings demonstrating a cystic immobile ovary adherent to the pelvic side wall. This case demonstrates that in women with unresolved ureteric compression where adnexal cyst is found, the investigator should look for features that suggest entrapment to the pelvic side wall.
\end{abstract}

Keywords Endometriosis - Ureteric obstruction · Ovarian cysts $\cdot$ Peritoneal pocket $\cdot$ Laparoscopy

\section{Introduction}

Endometriosois affects $2.5-15 \%$ of women of reproductive age. Ureteric involvement associated with endometriosis is rare and most often endometriosis is identified as the cause

A. G. Rajasri

Obstetrics and Gynaecology, Derriford Hospital,

Devon, PL6 8DH, UK

A. G. Rajasri $(\bowtie)$

8 Chartley, 22 The Avenue, Sneyd Park,

Bristol, BS9 1PE, UK

e-mail: rajasri_iyer@yahoo.com

Tel.: +44-117-9681846

Fax: +44-1452-395713

D. Byrne

Obstetrics and Gynaecology, Royal Cornwall Hospital,

Truro, UK of obstruction only during surgery. We report an interesting case of a trapped ovary causing extrinsic ureteric compression associated with endometriosis. The patient had longstanding ureteric colic, which was completely cured on removal of the right ovary found to be trapped in a peritoneal pouch.

\section{Case report}

A 33-year-old parous woman presented with right loin to groin pain, which had lasted for 18 months following the normal delivery of her fifth child. The pain was colicky, almost always present and associated with urgency of micturition. She had a regular 28-day menstrual cycle with heavy bleeding for 4-5 days. Clinically she was well except for mild right renal angle tenderness and right iliac fossa tenderness. She was referred as an emergency with exacerbation of the right loin pain and investigated under the urologists. No cause was found and cholecystitis and renal calculi were excluded. However, an intravenous urography (IVP) demonstrated mild obstruction of the right ureter with mild renal pelvicalyceal dilatation. The urologist arranged a CT scan to look for any lesions causing ureteric compression. The CT scan demonstrated mild ureteric dilatation within physiological limits, but, in addition, revealed a 3-cm right adnexal mass. After referral to the gynaecologists an ultrasound scan was carried out and a $3-\mathrm{cm}$ physiological cyst of the right ovary was confirmed. The gynaecologist reassured the woman that this was not a cause for concern. However, the pain persisted and the urologist advised a second gynaecological opinion. At this subsequent gynaecological examination bimanual palpation demonstrated an adnexal mass and pressure on this mass reproduced the loin pain. A transvaginal scan revealed an immobile tender right ovary with a simple cyst measuring $3.8 \times 3.3 \mathrm{~cm}$, adherent to the pelvic side wall. The uterus and the left ovary were normal. The provisional diagnosis was adnexal pathology, causing secondary ureteric compression. The patient underwent a diagnostic laparoscopy, which revealed a normal uterus and left ad- 


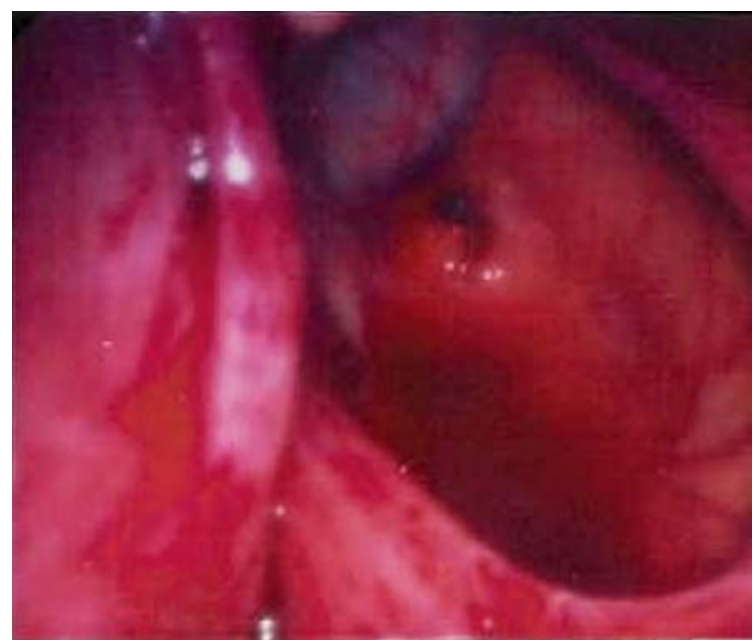

Fig. 1 The peritoneal defect in the broad ligament

nexa. The right ovary was trapped in a peritoneal defect in the broad ligament forming a pouch within the pelvic sidewall (Fig. 1). This trapped ovary was compressing the right ureter explaining the symptoms and the hydronephrosis. The ovary was delivered through the defect and the cyst was drained. Attempts to ensure the ovary remained outside the pouch failed and hence a right salpingo-oophorectomy was performed. The patient was immediately aware the pain had gone from the first postoperative day, confirming the adnexal cyst was the cause of her loin pain. At the time of surgery a single spot of endometriosis was seen at the base of the peritoneal pouch, it was presumed that the pouch might have been present due to endometriosis. The combined contraceptive pill was prescribed as a continuous medication for further treatment of the endometriosis. Surgical follow up at 6 weeks confirmed that the pain had not recurred.

\section{Discussion}

This case is the first report of a trapped ovary in a peritoneal pocket associated with endometriosis causing extrinsic ureteric compression. In most of the previous reports of ureteric involvement in endometriosis, the patient was known to suffer from endometriosis [1-3] and the ureteric involvement was due to periureteric fibrosis or direct mechanical compression by the cysts $[1,2,4,5]$. Often, endometriosis is not identified to be the cause of the obstruction before surgery [1-4]. In this case the diagnosis was suspected preoperatively from the clinical and trans- vaginal scan findings demonstrating a cystic immobile ovary adherent to the pelvic side wall. Laparoscopy revealed that the peritoneal defect was holding the ovary against the ureter and causing compression with secondary loin pain. The puckered pigmented lesion at the base of the defect confirmed the presence of endometriosis and may be the cause of the defect itself [6]. There was no evidence of extensive pelvic endometriosis. Ureteric involvement in endometriosis is very rare (1.2\%) [7] and most often extrinsic. Successful medical management of extrinsic ureteric involvement has been reported [1, 2]. Once fibrosis has occurred medical management is not effective at altering the course of obstruction [2]. In our case an oophorectomy was performed because the compression on the ureter was thought to be mechanical and the ovary could not be repositioned away from the defect.

Post-operatively the patient was totally symptom-free, which confirms the diagnosis, and she is on medical treatment to prevent recurrence or growth of endometriosis.

\section{Conclusion}

This case demonstrates that in any women with unresolved ureteric compression where an adnexal cyst is found, the investigator should look for features to suggest entrapment on the pelvic side wall. This patient endured 18 months of pain without a diagnosis being reached. The ovarian cyst was physiological as the first gynaecologist explained, but its immobility was missed. The consequence of this finding was eloquently shown at laparoscopy.

\section{References}

1. Deprest J, Marchal G, Brosens I (1997) Obstructive uropathy secondary to endometriosis. N Engl J Med 337:1174-1175

2. Brough RJ, O'Flynn K (1996) Recurrent pelvic endometriosis and bilateral ureteric obstruction associated with hormone replacement therapy. BMJ 312:1221-1222

3. Ryan JF, Booth CM (1992) Endometriosis of the ureter. Br J Urol 69:430-431

4. Sanyal D, Argent VP (2003) Silent pelvic endometriosis presenting as pyelonephritis and ureteric obstruction. J Obstet Gynaecol 23:328-329

5. Esen T, Akinci M, Ander H, Tunc M, Tellaloglu S, Narter I (1990) Bilateral ureteric compression secondary to endometriosis. Br J Urol 66:98-99

6. Walter JA, Hentz GJ, Magtibay MP, Cornella LJL, Magrina FJ (2001) Endometriosis: correlation between histologic and visual findings at laparoscopy. Am J Obstet Gynecol 184:1407-1413

7. Ball TL, Platt MA (1963) Urological complications of endometriosis. Am J Obstet Gynecol 84:1516-1521 